\title{
BOREL ORDERINGS
}

\author{
LEO HARRINGTON, DAVID MARKER AND SAHARON SHELAH
}

\begin{abstract}
We show that any Borel linear order can be embedded in an order preserving way into $2^{\alpha}$ for some countable ordinal $\alpha$ and that any thin Borel partial order can be written as a union of countably many Borel chains.
\end{abstract}

0. Introduction. We say that $\langle\mathbf{X}, \leq\rangle$ is a Borel order if $\mathbf{X}$ and $\leq$ are Borel subsets of $\mathbf{R}$ and $\mathbf{R}^{2}$ respectively and $\leq$ is reflexive and transitive. Friedman $[\mathbf{F}]$ initiated the study of Borel orders, suggesting that this was an interesting class of uncountable orderings which avoids many of the pathologies of the uncountable. A number of results in this direction were proved in $[\mathbf{F}, \mathbf{H}-\mathbf{S}$ and $\mathbf{S}]$. In this paper we will give several structure theorems for Borel orderings which, in some sense, explain why pathologies are avoided.

$\langle\mathbf{X}, \leq\rangle$ is said to be thin if there is no perfect set of pairwise incomparable elements. Orderings that are thin admit reasonable structure theorems. There is little to say about nonthin orderings. The main results are

THEOREM 3.1. If $\langle X, \leq\rangle$ is a thin Borel order, then for some $\alpha<\omega_{1}$ there is a Borel $f: \mathbf{X} \rightarrow 2^{\alpha}$ order preserving (where $2^{\alpha}$ is ordered lexicographically).

THEOREM 5.1. If $\langle X, \leq\rangle$ is a thin Borel order, then $\mathbf{X}$ can be written as a countable union of Borel chains.

These results were proved by the first and third authors. The second author added the results of $\S 4$.

To prove these results it is useful to look instead at the lightface (i.e. $\Delta_{1}^{1}$ ) refinements. The proofs rely on the interplay of effective descriptive set theory and forcing. The tools we need are developed in $\S 1$ and $\S 2$. Other descriptive settheoretic facts can be found in Moschovakis [M]. As usual in descriptive set theory we will interchange $\mathbf{R}$ and $\omega^{\omega}$ whenever convenient.

Notationally, if $\leq$ is an order, $x \approx y$ if $x \leq y$ and $y \leq x$ and $x \mid y$ if $x$ and $y$ are incomparable. Lower case latin letters usually denote reals (with $e, m, n, i, j$ reserved for integers). Upper case latin letters denote sets of reals and script letters denote sets of sets of reals.

1. The reflection lemmas. Let $W_{0}, W_{1}, W_{2}, \ldots$ be the usual enumeration of r.e. sets. Let $U \subseteq \omega \times \omega^{\omega}=\left\{(e, x): \forall y \exists n(x|n, y| n, n) \in W_{e}\right\}$. Then $U$ is $\omega$-universal $\Pi_{1}^{1}$. Let $U_{e}=\{x:(e, x) \in U\}$.

DEFINITION 1.1. If $\mathscr{A} \subset \mathscr{P}(\mathbf{R})$ we say $\mathscr{A}$ is $\Pi_{1}^{1}$ on $\Pi_{1}^{1}$ if $\left\{e: U_{e} \in \mathscr{A}\right\}$ is $\Pi_{1}^{1}$.

Received by the editors January 22, 1987 and, in revised form, August 19, 1987.

1980 Mathematics Subject Classification (1985 Revision). Primary 03E15; Secondary 04A15.

The first and second authors were supported by NSF grants, and the third author was supported by a NSERC grant. 
Lemma 1.2 (Reflection). If $\mathscr{A}$ is $\Pi_{1}^{1}$ on $\Pi_{1}^{1}$ and $Y \in \mathscr{A}$ is $\Pi_{1}^{1}$, then there is a $\Delta_{1}^{1} X \subseteq Y$ such that $X \in \mathscr{A}$.

Proof. Let $\Psi: U \rightarrow \omega_{1}$ be a $\Pi_{1}^{1}$-norm. Let $n, m \in \omega$ such that $\left\{e: U_{e} \in\right.$ $\mathscr{A}\}=U_{n}$ and $Y=U_{m}$. For $e \in \omega$ let $V_{e}=\{y \in Y: \Psi(m, y)<\Psi(n, e)\}$ (i.e.: $y \in V_{e} \Leftrightarrow y$ gets into $Y$, before $U_{e}$ gets into $\left.\mathscr{A}\right)$. Each $V_{e}$ is $\Pi_{1}^{1}$ and we can easily find a recursive function $f$ such that $V_{e}=U_{f(e)}$. By the recursion theorem there is an $\hat{e} \in \omega$ s.t. $W_{\hat{e}}=W_{f(\hat{e})}$. Then by the definition of $U$ and $f, V_{\hat{e}}=U_{f(\hat{e})}=U_{\hat{e}}$.

If $U_{\hat{e}} \notin \mathscr{A}$, then $\Psi(n, \hat{e})=\infty$ and $V_{\hat{e}}=Y$. But $Y \in \mathscr{A}$ and $V_{\hat{e}}=U_{\hat{e}}$, so this is a contradiction and $U_{\hat{e}} \in \mathscr{A}$. If $\alpha=\Psi(n, \hat{e})<\omega_{1}^{c k}$, then $V_{\hat{e}}=\{y: \Psi(m, y)<\alpha\}$ and this set is $\Delta_{1}^{1}$. Thus $U_{\hat{e}}$ is the desired set.

Definition 1.3. Let $\mathscr{A} \subset \mathscr{P}(\mathbf{R})^{2}$. We say $\mathscr{A}$ is $\Pi_{1}^{1}$ on $\Pi_{1}^{1}$ if $\left\{\left(e, e^{\prime}\right)\right.$ : $\left.\mathscr{A}\left(U_{e}, U_{e^{\prime}}\right)\right\}$ is $\Pi_{1}^{1}$. $\mathscr{A}$ is monotonic upward if whenever $\mathscr{A}(Y, Z), Y \subset Y^{\prime}$ and $Z \subset Z^{\prime}$, then $\mathscr{A}\left(Y^{\prime} Z^{\prime}\right)$. We say $\mathscr{A}$ is continuous downward if whenever we have $Y_{0} \supseteq Y_{1} \supseteq Y_{2} \supseteq \cdots$ and $Z_{0} \supseteq Z_{1} \supseteq Z_{2} \supseteq \cdots$ such that for all $n \mathscr{A}\left(Y_{n}, Z_{n}\right)$, then $\mathscr{A}\left(\cap Y_{n}, \cap Z_{n}\right)$.

Lemma 1.4 (Strong Reflection). If $\mathscr{A} \subseteq \mathscr{P}(\mathbf{R})^{2}$ is $\Pi_{1}^{1}$ on $\Pi_{1}^{1}$, monotonic upward and continous downward, then if $Y \in \Pi_{1}^{1}$ and $\mathscr{A}(Y, \neg Y)$, there is a $\Delta_{1}^{1} X \subseteq Y$ such that $\mathscr{A}(X, \neg X)$.

\section{PROOF.}

Claim. If $X \subseteq Y$ is $\Delta_{1}^{1}$, there is $\Delta_{1}^{1} \hat{X} \supseteq X$ such that $\mathscr{A}(\hat{X}, \neg X)$ and $\hat{X} \subseteq Y$.

Consider $B(Z)=\{Z: A(Z, \neg X)$ and $X \subseteq Z\}$. Then $B$ is $\Pi_{1}^{1}$ on $\Pi_{1}^{1}$. Since $\mathscr{A}$ is monotonic upward and $\neg Y \subseteq \neg X, B(Y)$. Thus by reflection there is a $\Delta_{1}^{1} \hat{X} \supseteq X$ such that $\mathscr{A}(\hat{X}, \neg X)$ and $\hat{X} \subseteq Y$.

Let $X_{0}$ be any $\Delta_{1}^{1}$ subset of $Y$. Given $X_{n}$ let $X_{n+1} \supseteq X_{n}$ be $\Delta_{1}^{1}$ such that $\mathscr{A}\left(X_{n+1}, \neg X_{n}\right)$ and $X_{n+1} \subseteq Y$. The procedure for going from $X_{n}$ to $X_{n+1}$ is uniform so $\left\langle X_{n}: n \in \omega\right\rangle$ is $\Delta_{1}^{1}$. Let $X=\bigcup X_{n}$. Then $X$ is $\Delta_{1}^{1}$ and $X \subseteq Y$. Since $\mathscr{A}\left(X_{n+1}, \neg X_{n}\right)$. By monotonicity for all $n \mathscr{A}\left(X, \neg X_{n}\right)$. Thus by continuity $\mathscr{A}\left(X, \cap \neg X_{n}\right)$. But $\neg X=\bigcap \neg X_{n}$, so $\mathscr{A}(X, \neg X)$.

There is a natural way for $\mathscr{A}$ 's that satisfy the hypothesis of strong reflection to arise. Let $P(\bar{x}, \bar{y})$ be $\Pi_{1}^{1}$. Let $\mathscr{A}(X, Y) \Leftrightarrow \forall \bar{x} \notin X \forall \bar{y} \notin Y P(\bar{x}, \bar{y})$. Clearly $\mathscr{A}$ is $\Pi_{1}^{1}$ on $\Pi_{1}^{1}$, monotonic upward and continuous downward. The following corollary gives the flavor of applications of strong reflection.

COROLlaRY 1.5. If $X$ is $\Sigma_{1}^{1}$ and linearly ordered by $\leq a \Delta_{1}^{1}$ ordering, then there is a $\Delta_{1}^{1} Y \supseteq X$ such that $Y$ is linearly ordered by $\leq$.

ProOF. Let $\mathscr{A}(Y, Z) \Leftrightarrow \forall x_{0}, x_{1}, \notin Y x_{0} \leq x_{1} \vee x_{1} \leq x_{0}$.

Then $\mathscr{A}$ satisfies the hypothesis of strong reflection and $\mathscr{A}(\neg X, X)$. So by strong reflection there is a $\Delta_{1}^{1} Z \subseteq \neg X$ such that $\mathscr{A}(Z, \neg Z)$. Let $Y=\neg Z$.

The reflection lemmas provide a uniform treatment for a number of results which otherwise would be proved by an ad hoc mix of $\Sigma_{1}^{1}$-separation and Kreisel uniformization arguments.

2. Gandy forcing. Let $\mathbf{P}=\left\{A \in \Sigma_{1}^{1}: A \neq \varnothing\right\}$. We order $\mathbf{P}$ by inclusion (i.e. $A \leq B$ iff $A \subseteq B$ ). This notion of forcing was used by Harrington $[\mathbf{H}]$ to give a new proof of Silver's theorem on $\Pi_{1}^{1}$ equivalence relations. 
LEMMA 2.1. If $G \subseteq \mathbf{P}$ is generic, then $\bigcap\{A: A \in G\}=\{a\}$ for some $a \in \mathbf{R}$.

Let $A \in G$ say $x \in A \Leftrightarrow \exists f R(f, x)$ where $R$ is $\Pi_{1}^{0}$. For $\sigma, \eta \in \omega^{\omega}$ let $A_{\sigma, \eta}=$ $\{x \supset \sigma: \exists f \supseteq \eta R(f, x)\}$. For each $n$ we can find $\sigma_{n} \eta_{n} \in \omega^{n}$ s.t. $A_{\sigma_{n}, \eta_{n}} \in G$ and $\sigma_{n} \subset \sigma_{n+1}, \tau_{n} \subset \tau_{n+1}$. Thus if $x=\lim _{n} \sigma_{n}$, and $f=\lim _{n} \eta_{n}$, then $R(f, x)$. For each $n\{y: y|n=x| n\} \in G$. Thus $\{x\}=\cap G$.

If $b \in \mathbf{R}$ we can view $b$ as coding a pair of reals $\left\langle b_{0}, b_{1}\right\rangle$.

LEMMA 2.2. If $b$ is $\mathbf{P}$-generic, then both $b_{0}$ and $b_{1}$ are $\mathbf{P}$-generic.

Proof. We show $b_{0}$ is $\mathbf{P}$-generic. Let $B \in \mathbf{P}$ and let $\mathscr{D} \subseteq \mathbf{P}$ be dense open. Let $B^{\prime}=\{x: \exists y(x, y) \in B\}$, since $B^{\prime} \in \mathbf{P}$ there is $C^{\prime} \subseteq B^{\prime}$ s.t. $C^{\prime} \in \mathscr{D}$. Let $C=\left\{(x, y): x \in C^{\prime} \wedge(x, y) \in B\right\}$. Then $C \neq \varnothing, C \subseteq B$ and $c \Vdash \stackrel{\circ}{b} \in C^{\prime}$. Thus $\mathscr{D}^{\prime}=\left\{C \in \mathbf{P}\right.$ : for some $\left.C^{\prime} \in \mathscr{D}, C \Vdash \stackrel{\circ}{b}_{0} \in C^{\prime}\right\}$ is dense, so $b_{0}$ is $\mathbf{P}$-generic.

In our main results we will be dealing with modified products of Gandy forcing.

Definition 2.3. $\mathbf{P}^{n}=\left\{A \subseteq \mathbf{R}^{n}: A\right.$ is $\Sigma_{1}^{1}$ and $\left.A \neq \varnothing\right\}$. Let $E$ be a $\Sigma_{1}^{1}$ equivalence relation. Let $\mathbf{P}_{E}^{n}=\left\{A \subseteq \mathbf{R}^{n}: A\right.$ is $\Sigma_{1}^{1}$ and if $\left(a_{1}, \ldots, a_{n}\right) \in A$, then $\left.\forall i, j \leq n a_{i} E a_{j}\right\}$. Let $\mathbf{P} \times{ }_{E} \mathbf{P}=\{(Y, A) \in \mathbf{P} \times \mathbf{P}:(Y \times A) \cap E \neq \varnothing\}$ and $\mathbf{P}_{E}^{2} \times{ }_{E} \mathbf{P}_{E}^{2}=\left\{(Y, A) \in \mathbf{P}_{E}^{2} \times \mathbf{P}_{E}^{2}: \exists\left(y_{0}, y_{1}\right) \in Y \exists\left(a_{0}, a_{1}\right) \in A y_{0} E y_{1} E a_{0} E a_{1}\right\}$. Each of these sets is ordered by inclusion.

LeMMA 2.4. If $b$ is $\mathbf{P}$-generic, then both $b_{0}$ and $b_{1}$ are $\mathbf{P}$-generic.

Proof. We show $b_{0}$ is $\mathbf{P}$-generic. Let $B \in \mathbf{P}$ and let $\mathscr{D} \subseteq \mathbf{P}$ be dense open. Let $B^{\prime}=\{x: \exists y(x, y) \in B\}$, since $B^{\prime} \in \mathbf{P}$ there is $C^{\prime} \subseteq B^{\prime}$ s.t. $C^{\prime} \in \mathscr{D}$. Let $C=\left\{(x, y): x \in C^{\prime} \wedge(x, y) \in B\right\}$. Then $C \neq \varnothing, C \subseteq B$ and $c \Vdash \stackrel{\circ}{b} \in C^{\prime}$. Thus $\mathscr{D}^{\prime}=\left\{C \in \mathbf{P}\right.$ : for some $\left.C^{\prime} \in \mathscr{D}, C \Vdash \stackrel{\circ}{b}_{0} \in C^{\prime}\right\}$ is dense, so $b_{0}$ is $\mathbf{P}$-generic.

In our main results we will be dealing with modified products of Gandy forcing.

Definition $2.3 \mathbf{P}^{n}=\left\{A \subseteq \mathbf{R}^{n}: A\right.$ is $\Sigma_{1}^{1}$ and $\left.A \neq \varnothing\right\}$. Let $E$ be a $\Sigma_{1}^{1}$ equivalence relation. Let $\mathbf{P}_{E}^{n}=\left\{A \subseteq \mathbf{R}^{n}: A\right.$ is $\Sigma_{1}^{1}$ and if $\left(a_{1}, \ldots, a_{n}\right) \in A$, then $\left.\forall i, j \leq n a_{i} E a_{j}\right\}$. Let $\mathbf{P} \times{ }_{E} \mathbf{P}=\{(Y, A) \in \mathbf{P} \times \mathbf{P}:(Y \times A) \cap E \neq \varnothing\}$ and $\mathbf{P}_{E}^{2} \times_{E} \mathbf{P}_{E}^{2}=\left\{(Y, A) \in \mathbf{P}_{E}^{2} \times \mathbf{P}_{E}^{2}: \exists\left(y_{0}, y_{1}\right) \in Y \quad \exists\left(a_{0}, a_{1}\right) \in A y_{0} E y_{1} E a_{0} E a_{1}\right\}$. Each of these sets is ordered by inclusion.

LEMMA 2.4. If $a, b$ are $\mathbf{P} \times{ }_{E} \mathbf{P}$ generic, then $a$ and $b$ are $\mathbf{P}$-generic.

Proof. Let $\mathscr{D} \subseteq \mathbf{P}$ be dense open, let $\langle A, B\rangle \in \mathbf{P} \times{ }_{E} \mathbf{P}$. Let $A^{\prime}=\{x \in A: \exists y \in$ $B x E y\}$. Let $C \in \bar{D}, C \subseteq A^{\prime}$. Then $\langle C, B\rangle \in \mathbf{P} \times_{E} \mathbf{P}$. Thus $\mathscr{D}^{\prime}=\{\langle C, X\rangle: C \in$ $\mathscr{D}\}$ is dense in $\mathbf{P} \times_{E} \mathbf{P}$. So $a$ is $\mathbf{P}$-generic.

LEMMA 2.5. If $\left(a_{0}, a_{1}\right),\left(b_{0}, b_{1}\right)$ are $\mathbf{P}_{E}^{2} \times_{E} \mathbf{P}_{E}^{2}$ generic, then each pair $\left(a_{i}, b_{j}\right)$ is $\mathbf{P} \times_{E} \mathbf{P}$ generic.

Proof. We will prove this for $a_{0}, b_{0}$. Let $\mathscr{D} \subseteq \mathbf{P} \times{ }_{E} \mathbf{P}$ be dense. Let $\langle A, B\rangle \in$ $\mathbf{P}_{E}^{2} \times_{E} \mathbf{P}_{E}^{2}$. Let $\bar{A}=\{x: \exists y x E y \wedge(x, y) \in A\}, \bar{B}=\{x: \exists y x E y$ and $(x, y) \in B\}$. Then $\langle\bar{A}, \bar{B}\rangle \in \mathbf{P} \times_{E} \mathbf{P}$. Let $\langle C, D\rangle \leq\langle\bar{A}, \bar{B}\rangle,\langle C, D\rangle \in \mathscr{D}$. Let $\hat{C}=\{(x, y) \in$ $A: x E y \wedge x \in C\}, \hat{D}=\{(x, y) \in B: x E y \wedge x \in D\}$. Since $\exists x_{0} \in C \exists x_{1} \in D x_{0} E x_{1}$, $x_{0} \in \bar{A}$ and $x_{1} \in \bar{B},\langle\hat{C}, \hat{D}\rangle \in \mathbf{P}_{E}^{2} \times_{E} \mathbf{P}_{E}^{2}$.

Thus $\mathscr{D}^{\prime}=\left\{\langle X, Y\rangle \in \mathbf{P}^{2} \times \mathbf{P}_{E}^{2}:\langle\{x: \exists y(x, y) \in X\},\{x: \exists y:(x, y) \in Y\}\rangle \in \mathscr{D}\right\}$ is dense. 
We would like to have that if $b_{0}, b_{1}$ are $\mathbf{P} \times_{E} \mathbf{P}$ generic then $b_{0} E b_{1}$. This seems unlikely in general but is true for an important class of equivalence relations $E$.

DEFINITION 2.6. We say $E$ is representable if for every $x, y$ if $x \not y$ then there is $\Delta_{1}^{1}$ set $X$ such that $x \in X, y \notin X$ and $\forall x \in X \forall y \notin X x \not y$.

LEMMA 2.7. If $E$ is representable and $a, b$ are $\mathbf{P} \times_{E} \mathbf{P}$ generic then $a E b$.

Proof. Suppose not. Let $\langle A, B\rangle \Vdash \stackrel{\circ}{a} \not \stackrel{\circ}{b}$. There is a $\Delta_{1}^{1}$ set $X$ such that $a \in X$, $b \notin X$ and for all $x \in X, y \notin X x \not L y$. Let $\hat{A}=A \cap X, \hat{B}=B-X$. Since $a, b$ are $\mathbf{P}$-generic, by Lemma $2.1\langle\hat{A}, \hat{B}\rangle \in \mathbf{P} \times_{E} \mathbf{P}$. But clearly $\langle\hat{A}, \hat{B}\rangle \notin \mathbf{P} \times_{E} \mathbf{P}$.

In applications we will be interested in finding perfect sets of mutually generic reals. To obtain $\mathbf{P} \times \mathbf{P}$ generic reals this is done much as it would be in Cohen forcing. The only difficulty arises if we reach a condition $A$ which is countable. By the effective perfect set theorem if $A \in \Sigma_{1}^{1}$ and $A$ is countable, then $A$ is a set of hyperarithmetic reals. In general we will only be doing forcing below conditions which do not contain any hyperarithmetic reals.

LEMMA 2.8. If $A \in \mathbf{P}$ and $|A|>\aleph_{0}$, then we can add a perfect set of mutually P-generic reals in $A$.

Proof. Let $\bar{A}=\left\{x \in A: x \notin \Delta_{1}^{1}\right\}$. Then $\bar{A} \in \mathbf{P}$ and we can do the usual arguments below $\bar{A}$.

Obtaining perfect sets of mutually $\mathbf{P} \times{ }_{E} \mathbf{P}$ generic reals is somewhat more delicate. We prove only the special case we need.

LEMMA 2.9. Let $\leq$ be a $\Delta_{1}^{1}$ ordering on $\mathbf{R}$. Suppose for each $A \in \mathbf{P}$ we have a $\Sigma_{1}^{1}$-equivalence relation $E_{A}$ such that if $B \subseteq A$, then $x E_{B} y \Rightarrow x E_{A} y$. Suppose there is a $Y$ s.t. for all $B \subseteq Y, B \in \mathbf{P}$ there are $b_{0}, b_{1} \in B$ such that $\left\langle b_{0}, b_{1}\right\rangle$ are $\mathbf{P} \times E_{B} \mathbf{P}$ generic and $b_{0}$ and $b_{1}$ are incomparable. Then there is a perfect subset of $Y$ of pairwise incomparable elements.

Proof. Let $\hat{\mathbf{P}}$ be the set of finite functions $\varphi: t \rightarrow\{B \in \mathbf{P}: B \subseteq Y\}$ s.t.

(i) $t \subseteq 2^{\omega}$ is a finite tree,

(ii) $\varphi(\varnothing)=Y$,

(iii) if $\eta \subseteq \tau \in t$, then $\varphi(\eta) \supseteq \varphi(\tau)$,

(iv) for $\eta^{\wedge} 0, \eta^{\wedge} 1 \in t\left\langle\varphi\left(\eta^{\wedge} 0\right), \varphi\left(\eta^{\wedge} 1\right)\right\rangle \Vdash_{E_{\varphi(\eta)}} \stackrel{\circ}{y}_{0}$ incomparable to $\stackrel{\circ}{y}_{1}$.

(v) Let $L_{t}=\{\eta \in t: \forall \tau \supset \eta \tau \notin t\}$, then there is $\left\langle a_{\eta}: \eta \in L_{t}\right\rangle$ s.t. $a_{\eta} \in \varphi(\eta)$ and if $\eta, \tau \in L_{t}$, then $a_{\eta} E_{\varphi(\eta \wedge \tau)} a_{\tau}(\eta \wedge \tau=\sigma$ where $\sigma \subseteq \eta, \tau$ and $\eta(|\sigma|) \neq \tau(|\sigma|))$. Order $\hat{\mathbf{P}}$ by extension. Suppose $\Psi: T \rightarrow \mathbf{P}$ is generic. Let $\hat{\Psi}:[T] \rightarrow \mathbf{R}$ by $\hat{\Psi}(f)=$ $\bigcap\{\Psi(f \mid n): n \in \omega\}$ it is routine to see that if $f, g \in[T]$, then $\hat{\Psi}(f)$ and $\hat{\Psi}(g)$ are $\mathbf{P} \times{ }_{E_{\psi(f \wedge g)}} \mathbf{P}$ generic and hence incomparable. We need only show that $T$ is perfect.

Suppose $\varphi: t \rightarrow \mathbf{P}$ is in $\hat{\mathbf{P}}$ and $\eta \in L_{t}$. Let $A_{\eta}=\left\{x \in \varphi(\eta): \exists\left\langle b_{\sigma}\right\rangle_{\sigma \in L_{t}} b_{\eta}=x\right.$ and $b_{\sigma} E_{\varphi(\sigma \wedge \tau)} b_{\tau}$ for all $\left.\sigma, \tau \in L_{t}\right\}$. Let $B_{0}, B_{1} \subseteq A_{\eta}, B_{0}, B_{1} \in \mathbf{P}$ s.t. $\left\langle B_{0}, B_{1}\right\rangle \Vdash \stackrel{\circ}{y}_{0}$ and $\stackrel{\circ}{y}_{1}$ are incomparable. Let $\hat{\varphi}: t \cup\left\{\eta^{\wedge} 0, \eta^{\wedge} 1\right\} \rightarrow \mathbf{P}$ s.t. $\hat{\varphi} \supseteq \varphi$ and $\hat{\varphi}\left(\eta^{\wedge} i\right)=B_{i}$. Then $\hat{\varphi} \in \hat{\mathbf{P}}$. Thus if $\Psi: T \rightarrow \mathbf{P}$ is generic, $T$ is perfect. 


\section{Representing $\Delta_{1}^{1}$ orderings.}

THEOREM 3.1. If $\langle\mathbf{X} \leq\rangle$ is a thin $\Delta_{1}^{1}$ order, then there is $\alpha<\omega_{1}^{c k}$ and a $\Delta_{1}^{1}$ order preserving $f: \mathbf{X} \rightarrow 2^{\alpha}$.

ProOF. Without loss of generality $\mathbf{X}=\mathbf{R}$.

Let $\mathscr{F}=\left\{f: \mathbf{R} \rightarrow 2^{\alpha}: \alpha<\omega_{1}^{c k}, f \in \Delta_{1}^{1}\right.$ and $\left.x \leq y \Rightarrow f(x) \leq f(y)\right\}$.

Claim $1 . \mathscr{F}$ is $\Pi_{1}^{1}$.

$n=\left\langle n_{0}, n_{1}\right\rangle$ codes an element of $\mathscr{F}$,

(1) $n_{0}$ codes a recursive ordinal $\alpha$,

(2) $n_{1}$ is a $\Delta_{1}^{1}$ code for a subset $G$ of $\mathbf{R} \times 2^{\alpha}$,

(3) $\forall x \exists y \in \Delta_{1}^{1}(x)(G(x, y) \wedge \forall z(G(x, z) \rightarrow z=y))$ and

(4) $\forall x, y, z, w\left((x \leq y \wedge G(x, z) \wedge G(y, w)) \rightarrow x \leq w\right.$ [in $\left.\left.2^{\alpha}\right]\right)$.

Since the quantifier $\exists y \in \Delta_{1}^{1}(x)$ is really universal, this is easily seen to be $\Pi_{1}^{1}$.

For $F \in \mathscr{F}$ we define an equivalence relation $E_{F}$ by $x E_{F} y$ if and only if $F(x)=$ $F(y)$. Let $E=\bigcap\left\{E_{F}: F \in \mathscr{F}\right\}$. Then $x E y \Leftrightarrow \forall F \in \Delta_{1}^{1}(F \in \mathscr{F} \Rightarrow F(x)=F(y))$. Since $\mathscr{F}$ is $\Pi_{1}^{1}$ and $\forall F \in \Delta_{1}^{1}$ is really existential, $E$ is a $\Sigma_{1}^{1}$-equivalence relation.

Our main claim is that $E=\approx$. Before establishing the main claim we will show how to establish the theorem from it. For the moment assume $E=\approx$.

Consider the following $\mathscr{A} \subseteq \mathscr{P}(\mathbf{R}), \mathscr{A}(X) \Leftrightarrow \forall x, y\left(x \not y \rightarrow \exists F \in \Delta_{1}^{1} F \in X\right.$, $F \in \mathscr{F}$ and $F(x) \neq F(y))$. Then $\mathscr{A}$ is $\Pi_{1}^{1}$ on $\Pi_{1}^{1}$ and $\mathscr{A}(\mathscr{F})$ so by reflection there is a $\Delta_{1}^{1} X \subseteq \mathscr{F}$ s.t. $\left.\forall x, y x \not y \rightarrow \exists F \in X F(x) \neq F(y)\right)$.

Let $F_{0}, F_{1}, F_{2}, \ldots$ be a $\Delta_{1}^{1}$ enumeration of $X$. Define $F_{0}^{*}, F_{1}^{*}, \ldots$ as follows.

(i) $F_{0}^{*}=F_{0}$.

(ii) Suppose $F_{n}^{*}: \mathbf{R} \rightarrow 2^{\gamma}$ and $F_{n+1}: \mathbf{R} \rightarrow 2^{\alpha}$. Define $F_{n+1}^{*}: \mathbf{R} \rightarrow 2^{\alpha+\gamma}$ by $F_{n+1}^{*}(x)=F_{n}^{*}(X)^{\frown} F_{n+1}(x)$.

It is easily seen that $F_{n+1}^{*}$ is order preserving and if $F_{i}(x) \neq F_{i}(y)$ for any $i \leq n+1$, then $F_{n+1}^{*}(x) \neq F_{n+1}^{*}(y)$. Let $F^{*}=\lim _{n} F_{n}^{*}$. Then $F^{*}$ is $\Delta_{1}^{1}$ (note: By boundedness we can find $\delta<\omega_{1}^{c k}$ s.t. for all $n F_{n}: \mathbf{R} \rightarrow 2^{\alpha}$ for some $\alpha<\delta$. Thus $F^{*}: \mathbf{R} \rightarrow 2^{\alpha}$ for some $\left.\alpha \leq \delta \cdot \omega\right)$ and if $x \not y, F^{*}(x) \neq F^{*}(y)$. Thus $F$ is the desired function. Thus we need only establish that $E=\approx$.

Let $Z=\{x: \exists y x E y \wedge x \not y\}$. Clearly $Z$ is $\Sigma_{1}^{1}$. If $Z=\varnothing$, then $E=\approx$, so we assume for purposes of contradiction that $Z \neq \varnothing$. Let $\mathbf{P}=\left\{A \in \Sigma_{1}^{1}: A \neq \varnothing\right.$ and $A \subseteq Z\}$.

Claim 2. If $a$ and $b$ are $\mathbf{P} \times{ }_{E} \mathbf{P}$ generic, then it is not the case that $a<b$ (or symmetrically it is not the case that $b<a$.).

Let $\langle A, B\rangle \in \mathbf{P} \times_{E} \mathbf{P}$ s.t. $\langle A, B\rangle \Vdash \stackrel{\circ}{a}<\stackrel{\circ}{b}$.

Case 1. $\forall a \in A \forall b \in B(a E b \Rightarrow a \nsupseteq b)$.

Let $A_{0}=\{x: \exists y \in A y E x \wedge y \geq x\}$. Let $B_{0}=\{x: \exists y \in B \quad y E x \wedge y \leq x\}$. Now $A_{0}$ and $B_{0}$ are $\Sigma_{1}^{1}$. $A_{0}$ is the downward closure of $A$ in each $E$ class and $B_{0}$ is the upward closure of $B$ in each $E$-class. Clearly $A_{0}$ and $B_{0}$ are disjoint. Let $\mathscr{A}(X, Y) \Leftrightarrow \forall x, y(x \notin X \wedge y \notin Y \rightarrow x \not \mathbb{E} y \vee y \not x) \wedge \forall z\left(z \notin X \rightarrow z \notin B_{0}\right)$. Then $\mathscr{A}$ satisfies the requirements for strong reflection and $\mathscr{A}\left(\neg A_{0}, A_{0}\right)$. Thus there is a $\Delta_{1}^{1} C \supseteq A_{0}$ s.t. $\mathscr{A}(\neg C, C)$. Thus $C$ is downward closed and disjoint from $B_{0}$.

Subclaim. There is $F^{*} \in \mathscr{F}$ s.t. $\forall x, y\left(\left(x E_{F} * y \wedge y \leq x \wedge x \in C\right) \rightarrow y \in C\right)$.

Let $\mathscr{A}(X) \Leftrightarrow \forall x, y\left(\left(\exists f \in \Delta_{1}^{1} f \in X \wedge f(x) \neq f(y)\right) \vee y \not x \vee x \notin C \vee y \in C\right)$. Then $\mathscr{A}$ is $\Pi_{1}^{1}$ on $\Pi_{1}^{1}$ and $\mathscr{A}(\mathscr{F})$. Thus by reflection there is a $\Delta_{1}^{1} X \subseteq \mathscr{F}$ s.t. 
$\mathscr{A}(X)$. As above we can build $F^{*} \in \mathscr{F}$ such that $x E_{F^{*}} y$ iff for all $F \in X x E_{F} y$. So $\forall x, y\left(\left(x E_{F *} y \wedge y \leq x \wedge x \in C\right) \rightarrow y \in C\right)$.

Suppose $F^{*} \mathbf{R} \rightarrow 2^{\alpha}$. Let $G: \mathbf{R} \rightarrow 2^{\alpha+1}$ by

$$
G(X)= \begin{cases}F^{*}(x)^{\wedge} 0, & x \in C, \\ F^{*}(x)^{\wedge} 1, & x \notin C .\end{cases}
$$

It is easy to see that $G \in \mathscr{F}$ and if $x E_{F^{*}} y, x \in C$ and $y \notin C$, then $x \not_{G} y$. In

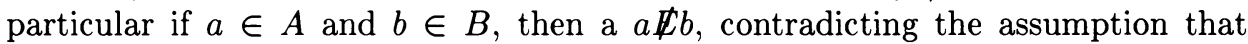
$\langle A, B\rangle \in \mathbf{P} \times{ }_{E} \mathbf{P}$.

Case 2. There are $a \in A, b \in B$ s.t. $a E b$ and $b \leq a$.

Let $D=\{(a, b): a \in A, b \in B, a E b$ and $b \leq a\} \in \mathbf{P}_{E}^{2}$. Let $\left(a_{1}, b_{1}\right),\left(a_{2}, b_{2}\right)$ be $\mathbf{P}_{E}^{2} \times_{E} \mathbf{P}_{E}^{2}$ generic such that $\left(a_{1}, b_{1}\right),\left(a_{2}, b_{2}\right) \in D$. By $2.5\left(a_{1}, b_{2}\right)$ and $\left(a_{2}, b_{1}\right)$ are each $\mathbf{P} \times{ }_{E} \mathbf{P}$ generic, so since $a_{i} \in A, b_{i} \in B, a_{1}<b_{2}$ and $a_{2}<b_{1}$. But $a_{1} \geq b_{1}$ and $a_{2} \geq b_{2}$ so $a_{1}<a_{1}$ a contradiction.

This completes the proof of Claim 2 .

Claim 3. If $a$ and $b$ are $\mathbf{P} \times{ }_{E} \mathbf{P}$ generic, then it is not the case that $a \approx b$.

Suppose $\langle A, B\rangle \in \mathbf{P} \times_{E} \mathbf{P}$ and $\langle A, B\rangle \Vdash \stackrel{\circ}{a} \approx \stackrel{\circ}{b}$.

Case 1. $\exists a \in A, b \in B a E b \wedge a<b$ (or $\exists a \in A b \in B a E b \wedge b<a$ ).

Let $D=\{(a, b): a \in A, b \in B, a E b$ and $a<b\}$. Let $\left(a_{0}, b_{0}\right),\left(a_{1}, b_{1}\right)$ be $\mathbf{P}_{E}^{2} \times{ }_{E} \mathbf{P}_{E}^{2}$ generic with both generic filters containing $D$. Then by $2.5\left(a_{0}, b_{1}\right)$ and $\left(a_{1}, b_{0}\right)$ are $\mathbf{P} \times_{E} \mathbf{P}$ generic. Thus $a_{0}<b_{0} \approx a_{1}<b_{1} \approx a_{0}$ a contradiction.

Case 2. $\exists a \in A, b \in B a E b \wedge a \mid b$.

Let $D=\{(a, b): a \in A, b \in B, a E b$ and $a \mid b\}$. Let $\left(a_{0}, b_{0}\right),\left(a_{1}, b_{1}\right)$ and $\left(a_{2}, b_{2}\right)$ be mutually $\mathbf{P}_{E}^{2} \times{ }_{E} \mathbf{P}_{E}^{2}$ generic with all three generic filters containing $D$. Then $a_{0} \mid b_{0}$ and by $2.5\left(a_{2}, b_{0}\right),\left(a_{2}, b_{1}\right)$ and $\left(a_{1}, b_{1}\right)$ are $\mathbf{P} \times{ }_{E} \mathbf{P}$ generic. Thus $b_{0} \approx a_{2} \approx b_{1} \approx a_{0}$. So we have a contradiction.

Case 3. $\forall a \in A \forall b \in B a E b \Rightarrow a \approx b$.

Let $C=\{x: x \in A \wedge \exists y \in B x E y\}$. Then $C$ is $\Sigma_{1}^{1}$ and $\forall x, y \in C(x E y \Rightarrow x \approx y)$. Since $A \subseteq Z, C \subseteq Z$. Thus $\forall x \in C \exists y(x E y \wedge x \not y)$. We claim this is impossible.

Subclaim. Let $X$ be $\Sigma_{1}^{1}$. Suppose $\forall x, y \in X(x E y \Rightarrow x \approx y)$. Then $\forall x \in X \forall y$ $(x E y \Rightarrow x \approx y)$.

Suppose not. Let $B^{+}=\{x: \exists y \in X x E y \wedge(x>y \vee x \mid y)\}$ and $B^{-}=\{x: \exists y \in X$ $x E y \wedge(x<y$ veex $\mid y)\}$. One of these is nonempty assume it is $B^{+}$. Let $A_{0}=$ $\{x: \exists y \in X x E y \wedge x \leq y\}$. Since $E / X=\approx, A_{0} \cap B^{+}=\varnothing$. As in Case 1 of Claim 3 by strong reflection we can find a $\Delta_{1}^{1} C$ s.t. $A_{0} \subseteq C, B^{+} \cap C=\varnothing$ and $C$ is downward closed in each $E$ class it intersects. By a second reflection argument we can find an $F \in \mathscr{F}$ s.t. if $x \in C \wedge x E_{F} y \wedge x \geq y$, then $y \in C$. Letting

$$
G(x)= \begin{cases}F(x)^{\wedge} 0, & x \in C, \\ F(x)^{\wedge} 1, & x \notin C .\end{cases}
$$

We see that if $x E_{F} y$ and $x \in A_{0}$ and $y \in B^{+}$, then $x \not \not_{G} y$, a contradiction. This establishes the subclaim and Claim 3 .

Thus if $a$ and $b$ are $\mathbf{P} \times_{E} \mathbf{P}$ generic, they are incomparable in $\leq$. By 2.9 it is possible to add a perfect set of mutually $\mathbf{P} \times_{E} \mathbf{P}$ generic elements. But then in the generic extension there is a perfect set of pairwise incomparable elements. This is $\Sigma_{2}^{1}$ so by Shoenfield absoluteness, there is already a perfect set of pairwise incomparable elements. Thus $\leq$ is not thin, a contradiction. 
COROLLARY 3.2 (HARRINGTON-SHELAH [HS]). If $\leq$ is a thin Borel order, there are no $\omega_{1}$-chains.

Theorem 3.1 is similar in spirit to the following result in mathematical economics.

THEOREM 3.3 (DEBREU [D]). If $(X, \leq)$ is a closed prelinear order then there is a continuous order preserving $f: X \rightarrow \mathbf{R}$.

\section{Separable $\Delta_{1}^{1}$ orders.}

DEFINITION 4.1. We say $(\mathbf{X}, \leq)$ is separable iff there is a countable $S \subseteq \mathbf{X}$ such that for any $x_{0}, x_{1} \in \mathbf{X}$ if $x_{0}<x_{1}$, there is $s \in S x_{0}<s<x_{1}$.

If $(\mathbf{X}, \leq)$ is a separable Borel prelinear order, then it is easy to see that there is a order preserving Borel $f: \mathbf{X} \rightarrow \mathbf{R}$. The main goal of this section is to prove the effective version of this result.

LEMMA 4.2. If $(\mathbf{X}, \leq)$ is a $\Delta_{1}^{1}$ prelinear order then either

(i) there is a perfect set of pairwise disjoint closed intervals $[a, b]$ with $a<b$ or

(ii) if $x_{0}<x_{1}$, there is a $\Delta_{1}^{1}$ downward closed $Y$ such that $x_{0} \in Y$ and $x_{1} \notin Y$.

PROOF. Without loss of generality $\mathbf{X}=\mathbf{R}$. We view each real $x$ as coding a pair $\left\langle x_{0}, x_{1}\right\rangle$. Let $A=\left\{x: x_{0}<x_{1} \wedge \forall Y \in \Delta_{1}^{1}[\forall z, y((z \leq y \wedge y \in Y) \rightarrow z \in Y)] \rightarrow\right.$ $\left.\left(x_{0} \notin Y \vee x_{1} \in Y\right)\right\}$. The quantifier $\forall Y \in \Delta_{1}^{1}$ is essentially existential so $A$ is $\Sigma_{1}^{1}$. Let $\mathbf{P}=\left\{B \in \Sigma_{1}^{1}: B \neq \varnothing\right\}$.

Claim 1. If $r \in \mathbf{R}$ and $\stackrel{\circ}{a}$ is a name for a generic real then $A \Vdash \neg\left(\stackrel{\circ}{a}_{0}<r<\stackrel{\circ}{a}_{1}\right)$.

Suppose not. Let $B \subseteq A$ such that $B \Vdash \stackrel{\circ}{a}_{0}<r<\stackrel{\circ}{a}_{1}$.

Case 1. There are $b, b^{\prime} \in B$ s.t. $b_{1} \leq b_{0}^{\prime}$.

Let $C=\left\{c: c_{0} \in B, c_{1} \in B \wedge c_{10} \geq c_{01}\right\}$. Let $c$ be $\mathbf{P}$ generic below $C$. Then by $2.2 c_{0}$ and $c_{1}$ are also $\mathbf{P}$ generic so $c_{00}<r<c_{01}$ and $c_{10}<r<c_{11}$. But $c \in C$ so $c_{01} \leq c_{10}$. Thus $r<r$ a contradiction.

Case 2. For all $b, b^{\prime} \in B b_{0}<b_{1}^{\prime}$.

Let $B^{-}=\left\{x: \exists y \in B \quad x \leq y_{0}\right\}$ and $B^{+}=\left\{x: \exists y \in B x \geq y_{1}\right\}$. Then $B^{-}$ and $B^{+}$are disjoint $\Sigma_{1}^{1}$ sets which are downward and upward closed respectively. Let $\mathscr{A}(X, Y) \Leftrightarrow \forall z \notin X z \notin B^{+} \wedge \forall x \notin X \forall y \notin Y y \not x$. Then $\mathscr{A}$ satisfies the hypothesis for strong reflection and $\mathscr{A}\left(\neg B^{-}, B^{-}\right)$. Thus by strong reflection there is a $\Delta_{1}^{1} C$ such that $B^{-} \subseteq C, C$ is downward closed and $C \cap B^{+}=\varnothing$. But then if $b \in B b_{0} \in C$ and $b_{1} \notin C$. Thus $B \nsubseteq A$ a contradiction.

Claim 2. If $r \in \mathbf{R}$, then $A \Vdash \stackrel{\circ}{a}_{0} \neq r$ and $\stackrel{\circ}{a}_{1} \neq r$. Suppose $B \in \mathbf{P}, B \subseteq A$ and $B \Vdash \stackrel{\circ}{a}_{0}=r$. If $b \in B$ and $b_{0} \neq r$, choose $n$ such that $b_{0}(n) \neq r(n)$. Then $B^{\prime}=\left\{c \in B: c(n)=b_{0}(n)\right\} \in \mathbf{P}$ and $B^{\prime} \Vdash \stackrel{\circ}{a}_{0}=r$. Thus for all $b \in B b_{0}=r$. But then $\{r\}=\left\{x: \exists b \in B \quad b_{0}=x\right\}$ is a $\Sigma_{1}^{1}$ singleton, so $r \in \Delta_{1}^{1}$. Then $Y=\{x: x \leq r\}$ is a downward closed $\Delta_{1}^{1}$ set separating $b_{0}$ from $b_{1}$ for $b \in B$, a contradiction.

Claim 3. If $a$ and $b$ are $\mathbf{P} \times \mathbf{P}$ generic, below $A$ then $\left[a_{0}, a_{1}\right] \cap\left[b_{0}, b_{1}\right]=\varnothing$.

This is clear from Claims 1 and 2 since $b$ is generic over $a$.

To prove the lemma we find a perfect set of mutually $\mathbf{P}$ generic reals below $A$. By Claim 3 these give rise to a perfect set of pairwise disjoint intervals.

THEOREM 4.3. If $(\mathbf{X}, \leq)$ is a $\Delta_{1}^{1}$ prelinear order, then either

(i) there is a perfect set of pairwise disjoint closed intervals $[a, b]$ with $a<b$, or

(ii) there is a $\Delta_{1}^{1} F: \mathbf{X} \rightarrow \mathbf{R}$ order preserving. 
Proof. Assume (i) fails. Let $\mathscr{F}=\left\{f: \mathbf{X} \rightarrow \mathbf{R}: f\right.$ is $\Delta_{1}^{1}$ and $x \leq y \Rightarrow f(x) \leq$ $f(y)\}$. As in $3.1 \mathscr{F}$ is $\Pi_{1}^{1}$.

Claim 1. If $x<y$, then there is $f \in \mathscr{F}$ s.t. $f(x)<f(y)$. By Lemma 4.2 there is a downward closed $Y \in \Delta_{1}^{1}$ such that $x \in y$ and $y \notin Y$. Let

$$
f(z)= \begin{cases}0, & z \in Y \\ 1, & z \notin Y .\end{cases}
$$

Clearly $f$ separates $x$ and $y$.

Claim 2. There is a $\Delta_{1}^{1} C \subseteq \mathscr{F}$ such that for all $x<y$ there if $f \in C$ such that $f(x)<f(y)$.

Let $\mathscr{A}(Z) \Leftrightarrow \forall x, y \in \mathbf{X}\left(x<y \rightarrow \exists f \in \Delta_{1}^{1} f \in Z \wedge f(x)<f(y)\right)$. Clearly $\mathscr{A}$ is $\Pi_{1}^{1}$ on $\Pi_{1}^{1}$ and $\mathscr{A}(\mathscr{F})$, so by reflection there is a $\Delta_{1}^{1} C \subseteq \mathscr{F}$ such that $\mathscr{A}(C)$.

Let $f_{0}, f_{1}, f_{2}$ be a $\Delta_{1}^{1}$ enumeration of $C$. By composing $f_{n}$ with an isomorphism $h_{n}: \mathbf{R} \rightarrow\left(1 / 2^{n+1}, 1 / 2^{n}\right)$ we may assume $F_{n}: \mathbf{X} \rightarrow\left(1 / 2^{n+1}, 1 / 2^{n}\right)$. Let $F(x)=$ $\sum_{n=0}^{\infty} f_{n}(x)$. It is easy to see that $F$ is well defined and $\Delta_{1}^{1}$.

Suppose $x \leq y$, then for any $n \in \omega f_{n}(x) \leq f_{n}(y)$. Thus $\sum_{n=0}^{\infty} f_{n}(x) \leq$ $\sum_{n=0}^{\infty} f_{n}(y)$. If $x<y$, there is a least $n$ s.t. $f_{n}(x)<f_{n}(y)$. Clearly $\sum_{n \neq i} f_{i}(x) \leq$ $\sum_{n \neq i} f_{i}(y)$, so $F(x)=f_{n}(x)+\sum_{n \neq i} f_{i}(x)<f_{n}(y)+\sum_{n \neq i} f_{i}(y)=F(y)$. Thus $F$ is order preserving. $F$ is the desired function.

COROLlaRY 4.4. If $(\mathbf{X}, \leq)$ is a separable $\Delta_{1}^{1}$ prelinear order there is a $\Delta_{1}^{1}$ $f: \mathbf{X} \rightarrow \mathbf{R}$ order preserving.

COROLLARY 4.5 (FRIEDMAN $[\mathbf{F}]$ ). If $(\mathbf{X}, \leq)$ is a Borel prelinear order then $(\mathbf{X}, \leq)$ is separable or there is a perfect set of totally isolated points.

COROllary 4.6 (Friedman-Shelah [F, St]). There is no Borel Suslin line.

5. Decomposing thin $\Delta_{1}^{1}$ orders. The following result is an analog of Dilworth's result [Di] that every partial order of width $n$ can be written as a union of $n$ chains.

THEOREM 5.1. If $(\mathbf{X}, \leq)$ is a thin $\Delta_{1}^{1}$ prepartial order, then there are $\Delta_{1}^{1}$ chains $\left\langle\mathbf{X}_{n}: n \in \omega\right\rangle$ such that $\mathbf{X}=\bigcup_{n \in \omega} \mathbf{X}_{n}$.

Proof. Without loss of generality assume $\mathbf{X}=\mathbf{R}$. Let $Z=\left\{Y \in \Sigma_{1}^{1}\right.$ : $\leq$ is a prelinear ordering on $Y\}$. Let $W_{0}=\bigcup\{Y: Y \in Z\}$. If $Y \in Z$, then by 1.5 there is a $\Delta_{1}^{1} X \supseteq Y$ such that $X \in Z$. Thus $z \in W_{0} \Leftrightarrow \exists Y \in \Delta_{1}^{1}(\forall x, y \in Y$ $(x \leq y \vee y \leq x) \wedge z \in Y)$. So by familiar arguments $W_{0}$ is $\Delta_{1}^{1}$. Let $W=\neg W_{0}$. If $W=\varnothing$, then $\mathbf{X}$ is the union of countably many $\Delta_{1}^{1}$ chains. So we assume $W \neq \varnothing$. We will reach a contradiction if we can show there is a nonempty linearly orderly $\Sigma_{1}^{1} Y \subseteq W$.

For $Y \in \Sigma_{1}^{1}, Y \neq \varnothing, Y \subseteq W$, let $\mathscr{F}_{Y}=\left\{F \in \Delta_{1}^{1}: \exists \alpha<\omega_{1}^{c k} F: \mathbf{R} \rightarrow 2^{\alpha}\right.$ s.t. $\forall x, y \in Y F(x)<F(y) \rightarrow x<y\}$. For $F \in \mathscr{F}_{Y}$ let $x E_{F} y \Leftrightarrow F(x)=F(y)$ and $x E_{Y} y \Leftrightarrow \forall F \in \mathscr{F}_{Y} F(x)=F(y)$. As in 3.1 each $\mathscr{F}_{Y}$ is $\Pi_{1}^{1}$ and each $E_{Y}$ is $\Sigma_{1}^{1}$ and representable.

If $x, y \in Y$ and $x E_{Y} y$, then there is $F \in \mathscr{F}$ such that $F(x) \neq F(y)$. Thus $x$ and $y$ must be comparable. If for all $x, y \in Y \quad x E_{Y} y \Rightarrow x \approx y$, then $\leq$ linear orders $Y$, a contradiction. Thus for each $Y$ we may assume $\exists x, y \in Y\left(x E_{Y} y \wedge x \not y\right)$. 
Main claim. For $Y \subseteq W$ either (a) there are $a, b \in Y \mathbf{P} \times_{E_{Y}} \mathbf{P}$ generic with $a$ and $b$ incomparable or (b) $Y$ is linearly ordered. If (a) holds then by $2.9 \leq$ is not thin and if $b$ holds we reach a contradiction. Thus the main claim suffices. So far now we assume that any two $\mathbf{P} \times_{E_{Y}} \mathbf{P}$ generic elements of $Y$ are comparable.

Let $A=\left\{a \in Y: \exists b \in Y b E_{Y} a \wedge b \not a\right\}$. If $A=\varnothing, Y$ is linearly ordered so we may assume $A \neq \varnothing$.

Claim 1. If $b$ and $c$ are $\mathbf{P} \times_{E_{Y}} \mathbf{P}$ generic then $b \not c$.

Suppose $\left(B_{0}, C_{0}\right) \Vdash \stackrel{\circ}{b} \approx \stackrel{\circ}{c}$. Let $B=\left\{x \in B_{0}: \exists y \in C_{0} x E_{Y} y\right\} . C=\{x \in$ $\left.C_{0}: \exists y \in B x E_{Y} y\right\}$. Since $\left(B_{0}, C_{0}\right) \in \mathbf{P} \times_{E_{Y}} \mathbf{P} B$ and $C$ are nonempty.

Subclaim. $\forall b \in B \forall c \in C b E_{Y} c \Rightarrow c \approx b$.

Assume there are $b \in B, c \in C$, such that $b<c$ (the case $b>c$ is symmetric). Let $D=\left\{(b, c): b \in B, c \in C, b E_{Y} c\right.$ and $\left.b<c\right\}$. Let $\left(b_{0}, c_{0}\right),\left(b_{1}, c_{1}\right) \in D$ be $\mathbf{P}_{E_{Y}}^{2} \times_{E_{y}} \mathbf{P}_{E_{Y}}^{2}$ generic. Then $\left(b_{0}, c_{1}\right)$ and $\left(b_{1}, c_{0}\right)$ are $\mathbf{P} \times_{E_{Y}} \mathbf{P}$ generic so $b_{0}<c_{0} \approx$ $b_{1}<c_{1} \approx b_{0}$, a contradiction.

Thus for all $b, b^{\prime} \in B$ if $b E_{Y} b^{\prime}$, then $\exists c \in C b E_{Y} c E_{Y} b^{\prime}$. But then $b \approx c \approx b^{\prime}$ so $\forall b, b^{\prime} \in B b E_{Y} b^{\prime} \Rightarrow b \approx b^{\prime}$. Next consider $\left\{d \in Y: \exists b \in B b E_{Y} d\right.$ and $b, d$ are incomparable $\}$. This must be empty else we can find $b, d \in B \times D \mathbf{P} \times{ }_{E_{Y}} \mathbf{P}$ generic and incomparable, contradicting our assumption. Thus for any $d \in Y \quad b \in B$ if $b E_{Y} d$, then $b$ is comparable with $\left.d\right\}$. Let $B^{-}=\left\{x: \exists y \in B x E_{Y} y \wedge x \leq y\right\}$ and $B^{+}=\left\{x: \exists y \in B \quad x E_{Y} y \wedge x>y\right\}$. Since on $B E_{Y}=\approx, B^{+} \cap B^{-}=\varnothing$. Let $\mathscr{A}(U, V) \Leftrightarrow \forall u \notin U \forall v \notin V\left(u E_{Y} v \vee v>u\right)$. Then $\mathscr{A}\left(\neg B^{-}, B^{-}\right)$so by strong reflection we can find $\hat{B} \in \Delta_{1}^{1}$ such that $\hat{B} \supseteq B^{-}, \hat{B} \cap B^{+}=\varnothing$ and $\forall u \in \hat{B} \forall v \notin \hat{B}$ $u E_{Y} v \Rightarrow u<v$. Let $\mathscr{D}(U) \Leftrightarrow \forall x, y \in Y\left(\exists F \in \Delta_{1}^{1} F \in U F(x) \neq F(y) \vee x \notin\right.$ $\hat{B} \vee y \in \hat{B} \vee x<y$. Then $\mathscr{D}$ is $\Pi_{1}^{1}$ on $\Pi_{1}^{1}$ and $\mathscr{D}\left(\mathscr{F}_{Y}\right)$. Hence by reflection there is $X \subseteq \mathscr{F}_{Y}$ s.t. $X$ is $\Delta_{1}^{1}$ and for all $x, y \in Y(x \in \hat{B} \wedge y \notin \hat{B} \wedge x \nless y) \rightarrow \exists F \in X$ $F(x) \neq F(y)$. As in 3.1 we can find an $F^{*} \in \mathscr{F}_{Y}$ such that for all $x, y F^{*}(x) \neq F^{*}(y)$ iff there is an $F \in X$ such that $F(x) \neq F(y)$. Let

$$
G(x)= \begin{cases}F(x)^{\wedge} 0, & x \in \hat{B} \\ F(x)^{\wedge} 1, & x \notin \hat{B}\end{cases}
$$

Suppose $x, y \in Y$ if $G(x)<G(y)$, then either $F(x)<F(y)$, in which case $x<y$, or $F(x)=F(y)$ and $x \in \hat{B}$ and $y \notin \hat{B}$ so $x<y$. Thus $G \in \mathscr{F}_{Y}$. Moreover since $\hat{B} \cap B^{+}=\varnothing, G$ splits an $E_{Y}$ class, a contradiction.

Claim 2. If $(B, C) \Vdash \stackrel{\circ}{b}<\stackrel{\circ}{c}$, then $\forall b, c \in B \times_{E_{Y}} C b<c$. (We abbreviate this as $B<E_{Y} C$.)

Let $B^{\prime}=\left\{b: \exists c \in C b E_{Y} c \wedge b \nless c\right\}$ and assume $B^{\prime} \neq \varnothing$. Suppose $b_{0}, b_{1} \in$ $B^{\prime} x_{E_{y}} B^{\prime}$ and $\mathbf{P} \times_{E_{Y}} \mathbf{P}$ generic. By our assumption $b_{0} \leq b_{1}$ or $b_{1} \leq b_{0}$. By Claim $1 b_{0} \not b_{1}$. Assume $b_{0}<b_{1}$. Let $B_{0}, B_{1} \subseteq B^{\prime}$ s.t. $\left(B_{0}, B_{1}\right) \Vdash \stackrel{\circ}{b}_{0}<\stackrel{\circ}{b}_{1}$. Let $D=\left\{\left(b_{0}, c\right) \in B_{0} \times_{E_{Y}} C: b_{0} \nless c\right\}$. Let $\left(b_{0}, c\right), b_{1}$ be $\mathbf{P}_{E_{Y}}^{2} \times_{E_{Y}} \mathbf{P}$ generic with $\left(b_{0}, c\right) \in D, b_{1} \in B_{1}$. By $2.7\left(b_{0}, b_{1}\right)$ are $\mathbf{P} \times_{E_{Y}} \mathbf{P}$ generic so $b_{0}<b_{1}$. Further $c$ and $b_{1}$ are $\mathbf{P} \times_{E_{Y}} \mathbf{P}$ generic. Thus since $b_{1} \in B, b_{1}<c$. But then $b_{0}<b_{1}<c$ a contradiction. 
Suppose $(B, C) \Vdash \stackrel{\circ}{b}<\stackrel{\circ}{c}$. Then by Claim $2 B<_{E_{Y}} C$. Let $\bar{B}=\{x: \exists b \in B$ $\left.x E_{Y} b \wedge x \leq b\right\}$. Let $\mathscr{A}(U, V) \Leftrightarrow \forall u \notin U \forall v \notin V\left(u \not \mathbb{E}_{Y} v \vee v \npreceq u\right) \wedge \forall z \notin Z \forall y \in C$ $z<y$. Then $\mathscr{A}(\neg \bar{B}, \bar{B})$ so by strong reflection there is a $\Delta_{1}^{1} \hat{B} \supseteq \bar{B}$ s.t. $\hat{B} \cap C=\varnothing$ and $\hat{B}$ is downward closed in each $E_{Y}$ class it intersects.

Claim 3. $\forall x y\left(x \in \hat{B} \wedge y \notin \hat{B} \wedge x E_{Y} h\right) \rightarrow x<y$.

Suppose not. Let $D=\left\{d \notin \hat{B}: \exists b \in \hat{B} b E_{Y} d \wedge b \nless d \wedge d \in Y\right\}$. We can find $d_{0}, d_{1} \in D$ s.t. $d_{0}$ and $d_{1}$ are $\mathbf{P} \times_{E_{Y}} \mathbf{P}$ generic. By our assumption and Claim $1 d_{0}<d_{1}$ or $d_{1}<d_{0}$. Say $\left(D_{0}, D_{1}\right) \Vdash d_{0}<d_{1}$. By Claim $2 D_{0}<E_{Y} D_{1}$. Let $B_{1}=\left\{b \in \hat{B}: \exists d \in D_{1}\left(b E_{Y} d \wedge b \nless d_{1}\right)\right\}$. Let $\left(b, d_{0}\right) \in B \times_{E_{Y}} D_{0}$ be $\mathbf{P}$ generic. Then by assumption and Claim $1 b$ and $d_{0}$ are comparable. Since $\hat{B}$ is downward closed $b<b_{0}$. Thus for all $d_{1} \in D_{1}$ if $b E_{Y} d_{1}$, then $b<d_{0}<d_{1}$, a contradiction.

Thus $\hat{B}$ is $\Delta_{1}^{1}$, downward closed and if $\exists b \in \hat{B} y \in Y y E_{Y} b$ and $y \notin \hat{B} y>b$. Now by reflection arguments similar to those in Claim 1, we can find a $G \in \mathscr{F}_{Y}$ which splits some $E_{Y}$ class. This gives the contradiction which proves the main claim and the theorem.

Corollary 5.2 (ShelaH $[\mathbf{S}]$ ). If $(\mathbf{X}, \leq)$ is a Borel order and there is an uncountable set of pairwise incomparable elements, then there is a perfect set of pairwise incomparable elements.

\section{REFERENCES}

[D] G. Debreu, Continuity properties of Paretan utility, International Economic Review 5 (1964).

[Di] R. P. Dilworth, A decomposition theorem for partially ordered sets, Ann. of Math. 51 (1950).

[F] H. Friedman, Borel structures in mathematics, manuscript, Ohio State University, 1979.

[H] L. Harrington, A powerless proof of a theorem of Silver, handwritten notes, Univ. of California, Berkeley, 1976.

[H-S] L. Harrington and S. Shelah, Counting equivalence classes for co- $\kappa$-Souslin relations, Logic Colloquium '80, Eds., D. Van Dalen, D. Lascar and T. J. Smiley, North-Holland, 1982.

[M] Y. Moschovakis, Descriptive set theory, North-Holland, 1980.

[S] S. Shelah, On co- $\kappa$-Souslin relations, Israel J. Math. 47 (1984).

[St] C. Steinhorn, Borel structures and measure and category logic, Model Theoretic Logics, Eds., J. Barwise and S. Feferman, Springer-Verlag, 1985.

Department of Mathematics, University of California, Berkeley, CaliforNIA 94720

Department of Mathematics, University of Illinois at Chicago, Chicago, ILLINOIS 60680

Department of Mathematics, Hebrew University, Jerusalem, ISRAEL

Department of Mathematics, Simon Fraser University, Burnaby, B. C., CANADA V5A 1 S6

Department of Mathematics, Rutgers University, New Brunswick, New JERSEY 08903 